\title{
EM in the Barents Sea: strategic use of electromagnetic measurements in the process of license applications.
}

\author{
Stein Fanavoll, Mercia Silva, Fabio Giannino, EMGS
}

The Barents Sea represents tremendous geologic challenges in oil and gas exploration. This is illustrated by drilling of numerous disappointing wells, emphasizing the need for new exploration techniques. Previous CSEM experiences in the Barents Sea showed that EM can detect commercial hydrocarbon accumulations, while clearly non-economic accumulations do not give significant EM responses. Therefore resistivity measurements could be a valuable indicator of commercial volumes of oil and gas in the region, allowing a possible reduction in the number of dry and non-productive wells.

EMGS acquired CSEM data in 30 blocks in the Barents Sea prior to the $20^{\text {th }}$ licensing round in Norway. The data was gathered in Spring/Summer 2008 and consist of wide azimuth $3 \times 3 \mathrm{~km}$ scanning grids having adjacent receiver lines staggered in the inline position. One line for each block was densely sampled ( $1 \mathrm{~km}$ receiver spacing) providing data suitable for inversion.

A particularly high degree of difference between horizontal and vertical resistivity (anisotropy) made the Barents Sea EM data processing rather challenging, and required a survey-specific approach and a thorough understanding of the area geology. Consequently, 3D modelling was an essential tool as it helps evaluating the EM responses induced by background geometries.

Also, high quality of phase data is a critical issue when dealing with anisotropic environment as it can be essential for obtaining reasonable and realistic geological models capable of explaining the data. Furthermore, an initial phase data analysis can ideally indicate the depth and extent of a buried resistive body.

The Barents Sea Program has revealed a series of interesting EM anomalies proving the method's potential as a strategic tool in licensing rounds. The future of EM methods depends not only on adoption and implementation by the oil industry actors in their exploration workflow, but also on a deeper focus on geology for processing, understanding and interpretation of EM data. 